Purdue University Purdue e-Pubs

2015

\title{
The Effect of Relative Humidity on Dropwise Condensation Dynamics
}

\author{
J. E. Castillo \\ Purdue University \\ J.A. Weibel \\ Purdue University, jaweibel@purdue.edu \\ S V. Garimella \\ Purdue University, sureshg@purdue.edu
}

Follow this and additional works at: https://docs.lib.purdue.edu/coolingpubs

Castillo, J. E.; Weibel, J. A.; and Garimella, S V., "The Effect of Relative Humidity on Dropwise Condensation Dynamics" (2015). CTRC Research Publications. Paper 244.

http://dx.doi.org/http://dx.doi.org/10.1016/j.ijheatmasstransfer.2014.09.080

This document has been made available through Purdue e-Pubs, a service of the Purdue University Libraries. Please contact epubs@purdue.edu for additional information. 


\title{
The effect of relative humidity on dropwise condensation dynamics ${ }^{1}$
}

\author{
Julian E. Castillo, Justin A. Weibel, Suresh V. Garimella ${ }^{2}$ \\ Cooling Technologies Research Center, an NSF IUCRC \\ School of Mechanical Engineering and Birck Nanotechnology Center \\ Purdue University, 585 Purdue Mall, West Lafayette, IN 47907
}

\begin{abstract}
Dropwise condensation of atmospheric water vapor is important in multiple practical engineering applications. The roles of environmental factors and surface morphology/chemistry on the condensation dynamics need to be better understood to enable efficient water-harvesting, dehumidication, and other psychrometric processes. Systems and surfaces that may promote faster condensation rates and selfshedding of condensate droplets could lead to improved mass transfer rates and higher water yields in harvesting applications. In the present study, experiments are performed in a facility that allows visualization of the condensation process on a vertically oriented, hydrophobic surface at a controlled relative humidity and surface subcooling temperature. The distribution and growth of water droplets are monitored across the surface at different relative humidities $(45 \%, 50 \%, 55 \%$, and $70 \%)$ at a constant surface subcooling temperature of $15^{\circ} \mathrm{C}$ below the ambient temperature $\left(20^{\circ} \mathrm{C}\right)$. The droplet growth dynamics exhibits a strong dependency on relative humidity in the early stages during which there is a large population of small droplets on the surface and single droplet growth dominates over coalescence effects. At later stages, the dynamics of droplet growth is insensitive to relative humidity due to the dominance of coalescence effects. The overall volumetric rate of condensation on the surface is also assessed as a function of time and ambient relative humidity. Low relative humidity conditions not only slow the absolute rate of condensation, but also prolong an initial transient regime over which the condensation rate remains significantly below the steady-state value.
\end{abstract}

Keywords: dropwise condensation, relative humidity, growth dynamics, droplet distribution, hydrophobic

\footnotetext{
${ }^{1}$ Submitted for possible publication in International Journal of Heat and Mass Transfer, August 2014

${ }^{2}$ Corresponding author, Tel: +1 765494 5621. E-mail address: sureshg@purdue.edu.
} 


\section{Nomenclature}

$k \quad$ thermal conductivity $(\mathrm{W} / \mathrm{mK})$

$R \quad$ single droplet radius $(\mu \mathrm{m})$

$\langle R\rangle$ average radius $(\mu \mathrm{m})$

$R H \quad$ relative humidity $(\%)$

$V \quad$ volume of condensate on the surface (ml)

$t$ time (min)

$T$ temperature $\left({ }^{\circ} \mathrm{C}\right)$

\section{Greek}

$\varepsilon \quad$ surface coverage

$\mu \quad$ power-law exponent for droplet radius growth

\section{Subscripts}

$\begin{array}{ll}\text { sub } & \text { subcooling } \\ i i & \text { second regime of droplet growth } \\ i i i & \text { third regime of droplet growth } \\ A & \text { first sub-regime within } i i \\ B & \text { second sub-regime within } i i \\ 1 & \text { regime } i i_{A} \text { to } i i_{B} \text { transition } \\ 2 & \text { regime } i \text { to } i i i \text { transition } \\ 3 & \text { end of regime } i i i\end{array}$

\section{Introduction}

Condensation of atmospheric water vapor is exploited in water harvesting [1]-[2], dehumidification [3], distillation/desalination [4], building heating and cooling [5], and other engineered systems.

Enhancement of the mass transfer rate during condensation and facilitation of liquid removal from the surface can reduce operational cost and improve efficiency in these applications.

In the presence of purely water vapor, water condenses on a surface that has been cooled below the vapor saturation temperature. Depending on the wettability of the surface, the condensate may form a liquid film (wetting) or distinct droplets (non-wetting). Dropwise condensation is preferred over filmwise condensation due to the higher rates of heat and mass transfer that may be achieved [6]; however, even small amounts of non-condensable gas in the system causes the rate of heat transfer to decrease substantially in both filmwise [7][8] and dropwise [9] modes. In the case of condensation from humid air at ambient temperatures, which contains a small concentration of water vapor, water condenses on a surface that has been cooled below the saturation temperature corresponding to the partial pressure of the 
vapor (i.e., the dew-point temperature). During condensation of water from humid air, dropwise condensation exhibits lowered rates of heat transfer, which may be similar to that of filmwise condensation [10]. In order to further enhance condensation, condensate droplets must be promptly removed after nucleation on the surface. Surface functionalization and engineering has been investigated $[11],[12]$ to reduce the pinning forces between the droplets and the surface, and hence the departure size, compared to conventional smooth surfaces [13],[14]. On the other hand, the rate of condensation is also governed by external atmospheric conditions (viz., relative humidity, wind speed, and surface cooling). In order to predict and evaluate the performance of systems reliant on condensation, it is equally important to understand how these external conditions affect the dynamics of dropwise condensation.

The dynamics of condensation of water vapor from air into liquid droplets on a surface has been extensively characterized [15]-[21]. Three stages of droplet growth may be identified [16],[18]-[20]: (i) initially a uniform layer of small droplets grows only by direct condensation on the interfaces of the droplets; (ii) as the droplets grow, they coalesce and the distance between adjacent droplets increases with time; and (iii) small droplets appear in the exposed space between the droplets and the average surface coverage asymptotically increases to a constant value. Once the droplets reach a critical size, they slide off the surface and new droplets grow in the spaces that have been swept clear. A combination of experimental observations, scaling analyses, and numerical modeling of condensation on non-wetting surfaces [19] showed that during the second regime, the growth of single droplet radii can be described by the power law $R \sim t^{\mu}$, where $\mu=1 / 3$. The average droplet radius followed $\langle R\rangle \sim t^{\mu_{i i}}$ where $\mu_{i i}=1$ (under ideal conditions where the flux of water vapor molecules to the surface is uniform).

Experimentally reported values for $\mu_{i i}$ are typically lower than unity due to the difference in boundary conditions from this idealized condition. Prior experimental investigations have only been performed at near $100 \%$ relative humidity, and the dependence of these reported rates of growth on relative humidity has not been experimentally investigated in the literature.

In the present work, dropwise condensation experiments are performed at four relative humidities $(R H=45 \%, 50 \%, 55 \%$, and $70 \%)$ and a constant surface subcooling temperature $\left(\Delta T_{\text {sub }}=15{ }^{\circ} \mathrm{C}\right)$; the humid air is maintained at $20^{\circ} \mathrm{C}$ and ambient pressure. The condensation dynamics (i.e., the droplet growth and distribution) are characterized as a function of relative humidity. The dependence of these dynamics on the relative humidity is then used to explain the trends in the volumetric rate of condensation on the surface for the first generation of droplets that grow on a fresh, dry substrate.

\section{Experimental methods}




\subsection{Surface preparation}

The condensation experiments are performed on smooth silicon surfaces, coated with Teflon (AF 1600, Dupont) to render them hydrophobic. The silicon substrates were rinsed with isopropyl alcohol and ethanol for $5 \mathrm{~min}$ each, blown dry with nitrogen gas, and placed on a hot plate for $10 \mathrm{~min}$ at $90{ }^{\circ} \mathrm{C}$. The wafers were then spin-coated with $2 \%$ mass fraction solution of Teflon in FC-77 at $4500 \mathrm{rpm}$ and baked at $90^{\circ} \mathrm{C}$ for 40 min to eliminate the remaining solvent. This process yields a $\sim 4.1 \mu \mathrm{m}$-thick hydrophobic film coating on the silicon surface. The sample surface has a measured average roughness of $0.026 \mu \mathrm{m}$; the contact angle with water is $119.7 \mathrm{deg}$ as measured from side-view images of droplets placed on the substrate.

\subsection{Fluid conditioning system and condensation test section}

A schematic diagram of the experimental fluid conditioning system is shown in Figure 1. The experimental facility has two separate flow circuits: Circuit A-B consists of a chiller (ThermoFlex, 900 W, Thermo Scientific) that recirculates cooled water through a cold plate (CP12, Lytron) attached to the test section. Circuit C-G adiabatically mixes dry nitrogen gas with nitrogen saturated with water vapor at controlled mass flow rates to achieve a desired relative humidity in the test section. Dry nitrogen entering (C) is regulated to 60 psi. Two downstream mass flow controllers (FMQ 5400, 0-2 SLM, Omega) regulate the amount of dry gas that passes through the humidifier (D-F) or bypasses to the adiabatic mixer (E-F). After entering the test section, the gas-mixture relative humidity is continuously measured with a hygrometer (HX92AV-RP1, Omega). The hygrometer output signal provides feedback to a PID control loop programmed in LabVIEW. All signals are acquired using a data acquisition system (PCI-6120, National Instruments). The control system is capable of maintaining a relative humidity in a range from $20 \%$ to $75 \%$ with a measurement uncertainty of $\pm 2.5 \%$ relative humidity.

The condensation test section shown in Figure 2 maintains the test sample surface at a controlled subcooling temperature enclosed within the humidity-controlled chamber.

Figure 2 (a) shows an exploded view of the test section. All components are mounted to the cold plate, which provides a constant base temperature for the attached thermoelectric cooler (ZT8, Laird Technologies). The thermoelectric cooler and temperature controller (MTTC-1410, Laird Technologies) are used for fine adjustment of the sample surface temperature. An aluminum block is attached to the top side of the thermoelectric cooler. Double-sided carbon conductive tape (PELCO Image Tabs) is used to affix the sample on top of the aluminum block; special care was taken to attach the carbon tape consistently and avoid trapped air pockets in the interfaces. The thermoelectric cooler and the aluminum block assembly is pressed against the cold plate by a thermally insulating (polyether ether ketone, PEEK) shroud $(k=0.25 \mathrm{~W} / \mathrm{m} \mathrm{K})$. 
The test sample assembly is enclosed in a $10 \mathrm{~cm} \times 10 \mathrm{~cm} \times 7 \mathrm{~cm}$ acrylic chamber. The chamber is equipped with gas inlet/outlet ports, a condensate liquid drain, and two glass windows for viewing in directions normal (Figure 2(b)) and parallel to the sample surface. A perforated gas diffuser plate is placed at the bottom of the chamber, in between the inlet and outlet ports, to evenly distribute the flow into the chamber.

Four $0.5 \mathrm{~mm}$-diameter T-type thermocouples (TMTSS-M050, Omega) are placed flush with the top surface of the aluminum block in notches. The surface temperature of the sample is assumed to be approximately equal to the average of these four temperature measurements. The temperature inside the chamber is monitored with a rake of three thermocouples evenly spaced $5 \mathrm{~mm}$ apart, with the closest thermocouple being $10 \mathrm{~mm}$ from the surface. The reading from the thermocouple furthest away from the surface is taken as the temperature of the ambient gas mixture. The reported surface subcooling temperature is the difference between the ambient gas mixture temperature and the surface temperature. All thermocouple measurements are referenced to a constant-temperature bath (TRCIII, Omega) maintained at $0 \pm 0.1{ }^{\circ} \mathrm{C}$ and are calibrated before testing to achieve a measurement uncertainty of \pm 0.3 ${ }^{\circ} \mathrm{C}$.

\subsection{Experimental procedure and visualization}

Each condensation experiment is performed at a specified relative humidity and surface subcooling temperature. After attaching the sample to the aluminum block, the chamber is sealed and purged with a continuous stream of dry nitrogen gas for approximately $20 \mathrm{~min}$ until the relative humidity stabilizes at $10 \%$. Simultaneously, the chiller circulates coolant to cool down the entire test section until the temperature of the surface and the gas mixture inside the chamber stabilize at $\sim 20{ }^{\circ} \mathrm{C}$.

After this initial conditioning, the gas mixture flow circuit controller is turned on with the desired setpoint relative humidity. After $\sim 10 \mathrm{~min}$, the relative humidity measured inside the chamber reaches a steady value within $\pm 5 \%$ error relative to the set-point value. Subsequently, the thermoelectric cooler controller is turned on; after $3 \mathrm{~min}$, the temperature at the surface reaches a constant value within $\pm 2 \%$ of the set-point temperature. By this procedure, condensation only begins when the surface temperature drops below the dew-point temperature as controlled by the fast-response thermoelectric stage, and after the chamber relative humidity and ambient temperature have already been stabilized. Each experiment was performed using a fresh and dried sample to ensure consistent substrate characteristics between experiments; repeatability was verified by performing multiple experiments under nominally identical test conditions.

A sequence of snapshot images normal to the surface are acquired every $30 \mathrm{~s}$ at $150 \times$ magnification using a long-focal-distance zoom lens (VH-Z50L, Keyence) with in-line illumination. The camera 
(FASTCAM 1024-PCI, Photron) and test section are mounted to micrometer stages to allow easier focusing on the surface of the sample. The spatial resolution was calibrated by measuring the grid pattern size of a calibration target (R1L3S3P Grid Distortion Target, ThorLabs). At 150× magnification, the spatial resolution is $3.99 \pm 0.6 \mu \mathrm{m} /$ pixel.

An automated image post-processing script was developed in MATLAB to recognize and characterize the size of droplets on the surface. Figure 3 shows the processing steps for a representative image. The contrast-enhanced raw images (Figure 3(a)) are segmented to a binary image (Figure 3(b)). Unphysical internal boundaries caused by light reflection off the droplets are eliminated in a two-step process: the binary color scheme is first reversed and then each group of pixels that cannot be connected with the background (i.e., a black dot inside a white spot) is filled. Each remaining circle that corresponds to a droplet is tagged based on the Hough transform technique [22] for edge recognition (Figure 3(c)). The recognized circular boundaries are shown overlaid on the original image in Figure 3(d). The radius and location of each droplet allows for identification of the total number of droplets present in each image. Also, by using the tagged identity of each droplet, the algorithm is capable of tracking single droplet growth in a set of sequential images.

\section{Results and discussion}

\subsection{Droplet growth regimes}

A detailed description of the condensation dynamics for an illustrative experimental condition $(R H=$ $70 \%$ and $\Delta T_{\text {sub }}=15^{\circ} \mathrm{C}$ ) provides a framework for comparison across different relative humidities. The condensation dynamics of the different droplet growth regimes are quantitatively assessed based on evolution of droplet size distribution, surface coverage, and average radius of condensate droplets.

Figure 4 (a) shows histograms of the droplet size distribution on the surface with corresponding inset images of the droplets. Each selected image exhibits the unique characteristics within the different regimes. At the magnification and frame rate used for visualization in the experiments, we do not resolve a pure regime $(i)$ where coalescence is absent. Droplet coalescence events are always present in the field of view between successive frames, and therefore the observed behavior is considered to begin within regime $(i i)$. Regime $(i i)$ is divided into sub-regimes $\left(i i_{\mathrm{A}}\right)$ and $\left(i i_{\mathrm{B}}\right)$ on account of contrasting growth dynamics.

During regime $\left(i i_{A}\right)(t=1.5 \mathrm{~min})$ the surface is covered by a homogeneous pattern of small droplets with similar sizes in a narrow range of radii from $15 \mu \mathrm{m}$ to $25 \mu \mathrm{m}$. As time proceeds, these droplets grow through two mechanisms: direct condensation on their liquid-gas interfaces, and coalescence. At some 
critical transition time, a significant change in the growth dynamics is realized. After this transition to regime $\left(i i_{B}\right)$, at $12.5 \mathrm{~min}$, the mean value of the distribution shifts to a larger radius $(\sim 50 \mu \mathrm{m})$ and coalescence effects significantly broaden the distribution by three times compared to the previous regime. Coalescence events in this second sub-regime contribute to the nucleation of new droplets in areas that were occupied by the original droplets prior to coalescence. Figure 4 (b) shows a time series of images for an example coalescence of two droplets (dashed circles) that leads to the nucleation of a new smaller droplet. Since this new droplet grows in close proximity to the large, coalesced droplet, it soon thereafter merges into the large drop, as is shown in the last image in the time series.

In regime (iii) ( $t=50 \mathrm{~min}$ ), the average distance between the large coalesced droplets increases, leading to spontaneous nucleation of new droplets in the bare spaces between the larger droplets. These new droplets exist for a comparatively longer time before merging with neighboring droplets, and as a result, the size distribution splits into two characteristic groups of droplets: the first has a narrow distribution at a small radius $(\sim 25 \mu \mathrm{m})$ as characteristic of regime $\left(i i_{A}\right)$, and the second has a larger mean droplet radius with a wider variation in radius size as characteristic of regime $\left(i i_{B}\right)$. Such a bimodal distribution has been previously reported [23],[24], and the behavior seen in regime (iii) is generally consistent with observations in the literature.

Lastly, the larger droplets reach a critical size and slide off the surface by gravity. Small droplets nucleate in the freshly exposed area, initiating a new condensation cycle, and the peak in the bimodal distribution at small radii becomes more pronounced. Similar characteristics were observed during subsequent growth and sweeping cycles; however, time periodicity of the cycle cannot be assessed based on this global distribution pattern due to the coexistence of multiple generations of droplets within the same field of view.

\subsection{Growth of temporal-average radius}

The droplet pattern characteristics within each regime can also be described in terms of the evolution of the average droplet radius ( $\langle R\rangle$ ) and the surface coverage $(\varepsilon)$ with time, as shown in Figure 5 for a representative relative humidity. During regime $(i i)(t<40 \mathrm{~min})$ the average radius increases with time in accordance with a power law of the form $\left\langle R>\sim t^{\mu_{i i}}\right.$. In regime (iii), (40 $\left.\mathrm{min}<t<87.5 \mathrm{~min}\right)$ the average droplet radius and the surface coverage reach constant values at $\langle R\rangle \approx 117 \mu \mathrm{m}$ and $\varepsilon \approx 55 \%$, respectively. At the end of regime (iii), the droplets slide off the surface, and a new generation of droplets begins to grow.

The transition between each regime is quantitatively defined based on a distinct change in the average-radius growth behavior. Figure 6 shows a logarithmic plot for the evolution of the droplet 
average radius. The dashed lines overlaid on the plot indicate fits to power laws of the form $\left\langle R>\sim t^{\mu_{i i}}\right.$ over the durations of each regime. A distinct change in $\mu_{i i}$ determines the transitions.

A change in the power-law exponent within regime (ii) is clearly observed, and points to the need for subdivision of this regime. The transition occurs at $t_{1}=2 \mathrm{~min}$ when the power-law exponent sharply changes from $\mu_{i i, A}=0.27$ in regime $\left(i i_{A}\right)$ to $\mu_{i i, B}=0.60$ in regime $\left(i i_{B}\right)$. While this distinction of growth behavior within regime (ii) has not been previously observed in the literature for experiments performed at high relative humidity, some quantitative comparisons against prior observations may be drawn. Since the size distribution of droplets present on the surface during regime $\left(i i_{A}\right)$ is very narrow, the behavior of average radius growth in regime $\left(i i_{A}\right)$ is found comparable to previously reported values for single droplet growth during regime (ii) in the literature $(\mu \approx 1 / 3)$ [15],[17],[21]. The behavior of average radius growth for regime $\left(i i_{B}\right)$ can be directly compared to previously reported values for average radius growth in regime $(i i)$ in the literature $\left(\mu_{i i} \approx 0.75\right)$ [15]-[17]. This observed drastic difference in the radius growth behavior between regime $\left(i i_{A}\right)$ and $\left(i i_{B}\right)$ may therefore be attributed to the respective dominance of growth by direct condensation versus coalescence of droplets, as is investigated further in subsequent sections.

The transition into regime (iii) in Figure 6 occurs at $t_{2}=40 \mathrm{~min}$; as the droplets become dispersed and the frequency of coalescence events is reduced, the rate of radius growth by direct condensation on large droplets is comparatively small $\left(\mu_{i i i}=0.11\right)$. Finally, droplets sliding off the surfaces marks the end of regime (iii) at $t_{3}=87.5 \mathrm{~min}$.

\subsection{Effect of relative humidity on temporal-average radius}

These unique average-radius growth dynamics in regime (ii) are further assessed as a function of relative humidity ( $R H=45 \%, 50 \%, 55 \%$, and $70 \%)$ for a constant surface subcooling temperature $\left(\Delta T_{\text {sub }}\right.$ $=15^{\circ} \mathrm{C}$ ). Figure 7 shows a logarithmic plot of the average radius versus time for the four different relative humidity cases. Power-law fits to the data were used to assess the growth behavior, and to quantitatively determine the regime transition points $t_{1}$ and $t_{2}$. Figure 8 shows the dependence of the power-law exponents with relative humidity during regimes $\left(i i_{A}\right)$ and $\left(i i_{B}\right)$.

During regime $\left(i i_{A}\right)$, the power-law rate of growth exponent increases with relative humidity from $\mu_{i i, A}=0.12$ at $R H=45 \%$ to $\mu_{i i, A}=0.27$ at $R H=70 \%$. This is indicated by the increased slope at higher relative humidity in Figure 7. As a result, the time of the transition to regime $\left(i i_{B}\right)$, which occurs at a consistent average radius size, is delayed for lower relative humidity $\left(e . g\right.$., from $t_{1}=2.0 \mathrm{~min}$ at $R H=$ $70 \%$ to $t_{1}=6.5 \mathrm{~min}$ at $R H=45 \%$ ). Conversely, relative humidity has little influence on the 
condensation dynamics when coalescence appears to be the dominant mechanism in regime $\left(i i_{B}\right)$; variation in $\mu_{i i, B}$ is negligible for relative humidity ranging from $45 \%$ to $70 \%$. The following section analyzes single-droplet growth mechanisms to explain these trends observed with relative humidity, which indicate that the appearance and persistence of regime $\left(i i_{A}\right)$ is amplified at lower relative humidity.

\subsection{Single droplet growth}

Tracking the evolution of single condensate droplets on the surface confirms the predominant mechanisms leading to the contrasting trends in average radius growth within regime (ii). Figure 9 shows the radius of one representative droplet over time at a relative humidity of 50\%. Growth is characterized by periods of direct condensation on the droplet surface and intermittent coalescence events. Four coalescence events occur in the lifetime of the droplet shown in Figure 9, and are characterized by a sudden increase in the radius of the droplet. The periods of direct condensation are characterized by power-law growth with slower absolute rates of growth as the droplet radius increases. At early stages of growth, the step changes upon coalescence are a comparatively smaller percentage of the radius growth rate by direct condensation, and therefore direct condensation governs the average-radius growth dynamics. At later stages within regime (ii), these increments are comparatively larger and the growth by direct condensation is also slower at larger droplet radii. Droplet coalescence dominates the average radius growth dynamics at these later times within regime (ii).

The power-law exponent describing average radius growth was only observed to be dependent on relative humidity in regime $\left(i i_{A}\right)$, where single droplet growth by direct condensation is predominant. This suggests that the relative humidity influences the behavior of single-droplet growth within an array of densely packed droplets at the early stages of growth. Other studies have previously indicated that single droplet growth-law exponents are sensitive to boundary conditions that influence the pattern of vapor mass flow to the surface from the surroundings [18]. To assess the influence of relative humidity on the growth only by direct condensation on the droplet interfaces (excluding growth by coalescence), the condensation mass flux on the interfaces of the individual droplets is calculated based on the instantaneous rate of change of droplet radius (omitting step changes due to coalescence). Figure 10 shows a plot of the condensation mass flux on the interfaces of single droplets as a function of the droplet size for each relative humidity tested (averaged across all droplets present on the surface). In all of the cases, the mass flux decreases as the droplet size increases. This indicates the importance of the thermal resistance of the droplet at all relative humidities tested; larger droplets impose a larger thermal resistance between the solid substrate and liquid-vapor interface of the droplet, thereby effectively reducing the subcooling below ambient at the interface. However, a significant change in the behavior is noted for the lower values of relative humidity tested (45\% and $50 \%)$. The mass flux dependence on radius is weaker, 
suggesting that vapor species diffusion becomes an important rate-limiting transport mechanism at low relative humidity that could alter the vapor species concentration profile in the ambient, and accordingly the growth-law exponent. Figure 10 also clearly indicates the strong dependence of the absolute magnitude of the single droplet condensation mass flux on the relative humidity.

\subsection{Condensation rate behavior with time}

A primary concern of applications relying on dropwise condensation of water vapor may often be the volumetric rate of condensation on the surface. Figure 11(a) describes the characteristic evolution of the volumetric condensation rate on the surface based on a selected case, with the droplet growth regimes overlaid. The data presented have been smoothed using a moving-average filter to reduce the noise associated with calculating a time rate of change from discrete data points. In general, the condensation rate first decreases and then increases in time. The trends in overall rate of condensation on the surface can be explained by the size effects in single droplet growth (Figure 10) and the droplet size distribution on the surface (Figure 11(b)).

At early times in regime $\left(i i_{A}\right)(t=3 \mathrm{~min})$, a uniform population of small droplets primarily contribute to the overall condensation rate. The condensation rate decreases sharply as the average size of the droplets present on the surface increases $(t=6 \mathrm{~min}$ ), and is consistent with the single droplet size effect shown previously in Figure 10.

As time proceeds into growth regime $\left(i i_{B}\right)(t=10 \mathrm{~min})$, the average distance between the larger droplets increases, and coalescence events spawn new droplets with small radii, as was described above with reference to Figure 4 (b). While coalescence events that create larger droplets would otherwise be expected to reduce the condensation rate, this population of new droplets with $R \lesssim 50 \mu$ m yield a net increase in the rate of condensation on the surface. As time proceeds, the size difference between small and the large droplets widens and the average condensation rate stabilizes. At this stage, small droplets that nucleate have an even greater influence on the overall rate. Each coalescence event between large droplets spurs the nucleation of many small droplets that then causes large fluctuations in the condensation rate on the surface (e.g., $t=40 \mathrm{~min})$.

The evolution of the volumetric condensation rate is shown for all the relative humidities tested in Figure 12. In general, the condensation rate is faster for higher relative humidity. At higher relative humidity, the initial reduction in the condensation rate with time is less pronounced and exists for a shorter period (or is suppressed entirely in the case of $70 \%$ relative humidity). Also, as described above, a transition from the initially decreasing to an increasing condensation rate is determined by the nucleation and growth of small interstitial droplets after coalescence events. Higher relative humidities are able to support nucleation at lower interstitial spacing upon coalescence of smaller droplet radii [23], 
whereas nucleation of interstitial droplets is suppressed for longer periods at low relative humidity. Low relative humidities thereby prolong the period of single droplet-like growth behavior that has a reducing condensation rate with time. Understanding these interdependent transport mechanisms during condensation, and engineering surfaces/systems that eliminate such low-performance transients, is key to the deployment of systems that condense vapor at low relative humidity.

\section{Conclusions}

The growth of water droplets on a smooth hydrophobic surface was investigated at different ambient relative humidities. The characteristic behavior of the early stages of droplet growth, before surface coverage parameters reached constant values, were analyzed as a function of the relative humidity. The growth of the average droplet radius versus time was suitably described by a power law in an initial regime that was characterized by a narrow distribution of small droplets; average growth behaved similarly to single-droplet growth and the exponent of the power law was sensitive to relative humidity in this regime. In the subsequent regime, coalescence dominated the average-radius growth dynamics, which were insensitive to relative humidity. Further interrogation of single-droplet radius growth, after filtering out the contribution of coalescence effects, confirmed these dependencies on relative humidity.

A strong dependence of the droplet size on the condensation mass flux on the interfaces of single droplets was observed (higher rates at smaller droplet sizes). This size dependence of the condensation rate was used to explain the temporal evolution of the overall volumetric condensation rate on the surface. As a generation of droplets grows on the surface, the condensation rate decreases as the droplets grow and coalesce. This trend does not reverse until the distribution of droplets becomes sparse enough that coalescence events induce nucleation of new small droplets on the surface, which increase the net condensation rate. This behavior is shown to be highly sensitive to the relative humidity. The surface is more easily starved of water vapor in the droplet interstices at lower ambient relative humidity. This prolongs and amplifies the period of reduced condensation rates on the surface.

\section{Acknowledgments}

The support for this work of the Cooling Technologies Research Center (CTRC) an NSF Industry/University Cooperative Research Center at Purdue University is gratefully acknowledged. The first author acknowledges the financial support provided by the Colombian department for science, technology and innovation (Colciencias) and the Colombia-Purdue Institute (CPI). 


\section{References}

[1] R. V. Wahlgren, "Atmospheric water vapour processor designs for potable water production: A review," Water Research, vol. 35, no. 1, pp. 1-22, 2001.

[2] D. Milani, A. Abbas, A. Vassallo, M. Chiesa, and D. A. Bakri, "Evaluation of using thermoelectric coolers in a dehumidification system to generate freshwater from ambient air," Chemical Engineering Science, vol. 66, no. 12, pp. 2491-2501, 2011.

[3] K. Hong and R. L. Webb, "Performance of dehumidifying heat exchangers with and without wetting coatings," Journal of Heat Transfer, vol. 121, no. 4, pp. 1018-1026, 1999.

[4] S. Parekh, M. M. Farid, J. R. Selman, and Said Al-hallaj, "Solar desalination with a humidification-dehumidification technique: A comprehensive technical review," Desalination. vol. 160, pp. 167-186, 2004.

[5] L. Pérez-Lombard, J. Ortiz, and C. Pout, "A review on buildings energy consumption information," Energy and Buildings, vol. 40, no. 3, pp. 394-398, 2008.

[6] J.W. Rose, "Dropwise condensation theory and experiment: A review," Proceedings of the Institution of Mechanical Engineers, Part A: Journal of Power and Energy, vol. 216, no. 2, pp.115-128, 2002.

[7] E. M. Sparrow and S. H. Lin, "Condensation heat transfer in the presence of a noncondensable gas,” Journal of Heat Transfer, vol. 86, no. 3, pp. 430-436, 1964.

[8] E. M. Sparrow, W. J. Minkowycz, and M. Saddy, "Forced convection condensation in the presence of noncondensables and interfacial resistance," International Journal of Heat and Mass Transfer, vol. 10, no. 12, pp. 1829-1845, 1967.

[9] D. W. Tanner, D. Pope, C. J. Potter, and D. West, "Heat transfer in dropwise condensation at low steam pressures in the absence and presence of non-condensable gas," International Journal of Heat and Mass Transfer, vol. 11, no. 2, pp. 181-190, 1968.

[10] C. Bum-Jin, K. Sin, K. Min Chan, and M. Ahmadinejad, "Experimental comparison of film-wise and dropwise condensation of steam on vertical flat plates with the presence of air," International Communications in Heat and Mass Transfer, vol. 31, no. 8, pp. 1067-1074, 2004.

[11] J. B. Boreyko and C.-H. Chen, "Self-propelled dropwise condensate on superhydrophobic surfaces," Physical Review Letters, vol. 103, no. 18, 184501, 2009.

[12] R. Enright, N. Miljkovic, J. L. Alvarado, K. Kim, and J. W. Rose, "Dropwise Condensation on Micro- and Nanostructured Surfaces," Nanoscale and Microscale Thermophysical Engineering, vol. 18, no. 3, pp. 223-250, 2014. 
[13] P. Dimitrakopoulos and J. J. L. Higdon, "On the gravitational displacement of three-dimensional fluid droplets from inclined solid surfaces," Journal of Fluid Mechanics, vol. 395, pp. 181-209, 1999.

[14] H. Y. Kim, H. J. Lee, and B. H. Kang, "Sliding of liquid drops down an inclined solid surface," Journal of Colloid and Interface Science, vol. 247, no. 2, pp. 372-380, 2002.

[15] D. Beysens and C. M. Knobler, "Growth of breath figures," Physical Review Letters, vol. 57, no. 12, pp. 1433-1436, 1986.

[16] J. L. Viovy, D. Beysens, and C. M. Knobler, "Scaling description for the growth of condensation patterns on surfaces," Physical Review A, vol. 37, no. 12, pp. 4965-4970, 1988.

[17] D. Fritter, C. M. Knobler, and D. A. Beysens, "Experiments and simulation of the growth of droplets on a surface (breath figures)," Physical Review A, vol. 43, no. 6, pp. 2858-2869, 1991.

[18] A. Steyer, P. Guenoun, D. Beysens, and C. M. Knobler, "Growth of droplets on a substrate by diffusion and coalescence," Physical Review A, vol. 44, no. 12, pp. 8271-8277, 1991.

[19] D. Beysens, "The formation of dew," Atmospheric Research, vol. 39, no. 1-3, pp. 215-237, 1995.

[20] J. Blaschke, T. Lapp, B. Hof, and J. Vollmer, "Breath figures: nucleation, growth, coalescence, and the size distribution of droplets," Physical Review Letters, vol. 109, no. 6, 068701, 2012.

[21] M. Mei, B. Yu, M. Zou, and L. Luo, “A numerical study on growth mechanism of dropwise condensation," International Journal of Heat and Mass Transfer, vol. 54, no. 9-10, pp. 20042013, 2011.

[22] T. J. Atherton and D. J. Kerbyson, "Size invariant circle detection," Image and Vision Computing, vol. 17, no. 11, pp. 795-803, 1999.

[23] R. N. Leach, F. Stevens, S. C. Langford, and J. T. Dickinson, "Dropwise condensation: experiments and simulations of nucleation and growth of water drops in a cooling system," Langmuir, vol. 22, no. 21, pp. 8864-8872, 2006.

[24] A. M. Macner, S. Daniel, and P. H. Steen, "Condensation on surface energy gradient shifts drop size distribution toward small drops," Langmuir, vol. 30, no. 7, pp. 1788-1798, 2014. 


\section{List of figures}

Figure 1. Schematic diagram of nitrogen-water gas mixture and chilled water flow circuits.

Figure 2. (a) 3D CAD model of the test section exploded assembly, and (b) front view photograph of the chamber.

Figure 3. Example image subject to post-processing by (a) contrast enhancement, (b) binary segmentation, (c) color scheme reversal, elimination of unphysical reflections, and droplet tagging, and (d) circular droplet boundary detection ( $\boldsymbol{R H}=70 \% ; \Delta \boldsymbol{T}_{\text {sub }}=15{ }^{\circ} \mathrm{C}, t=15.5 \mathrm{~min}$ ).

Figure 4. (a) Histogram of number of droplets at a given size at $t=1.5,12.5,50$, and $90 \mathrm{~min}$. Inset images show condensed droplets on the substrate. (b) The set of bottom images depicts a coalescence event in regime $(i i B) .\left(\boldsymbol{R H}=70 \% ; \Delta \boldsymbol{T}_{\text {sub }}=15{ }^{\circ} \mathrm{C}\right.$. $)$

Figure 5. Average radius (left axis) and surface coverage (right axis) versus time for $\boldsymbol{R H}=70 \%$ and $\Delta \boldsymbol{T}_{\text {sub }}=15^{\circ} \mathrm{C}$.

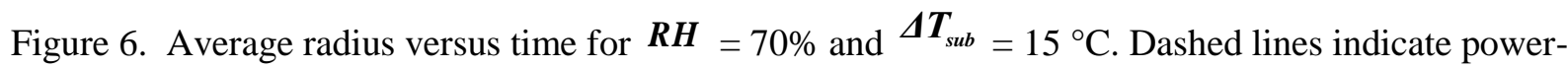
law fits within each regime.

Figure 7. Average radius versus time at $\boldsymbol{\Delta \boldsymbol { T } _ { \text { sub } }}=15^{\circ} \mathrm{C}$ and $70 \%, 55 \%, 50 \%$, and $45 \%$ relative humidity.

Figure 8. Exponent of the power-law fit to the temporal average radius data of the form $\langle\boldsymbol{R}\rangle \sim \boldsymbol{t}^{\mu}$ for regime $(i i A)\left(\boldsymbol{\mu}_{i i, A}\right)$ and regime $(i i B)\left(\boldsymbol{\mu}_{i i, B}\right)$ as a function of relative humidity.

Figure 9. Radius versus time for a single droplet at $\boldsymbol{\Delta \boldsymbol { T } _ { \text { sub } }}=15^{\circ} \mathrm{C}$ and $50 \%$ relative humidity. Inset images show a set of droplets before and after the last coalescence event.

Figure 10. Rate of condensation per unit surface area of a single droplet as a function of droplet radius at $\boldsymbol{\Delta} \boldsymbol{T}_{\text {sub }}=15^{\circ} \mathrm{C}$ and $70 \%, 55 \%, 50 \%$, and $45 \%$ relative humidity.

Figure 11. (a) Volumetric rate of condensation on the surface as function of time, and (b) time series of images of the droplets on the surface for ${ }^{\Delta \boldsymbol{T}_{s u b}}=15^{\circ} \mathrm{C}$ and $50 \%$ relative humidity.

Figure 12. Volumetric rate of condensation on the surface as a function of time at $\boldsymbol{\Delta \boldsymbol { T } _ { \text { sub } }}=15^{\circ} \mathrm{C}$ and $70 \%, 55 \%, 50 \%$, and $45 \%$ relative humidity. 


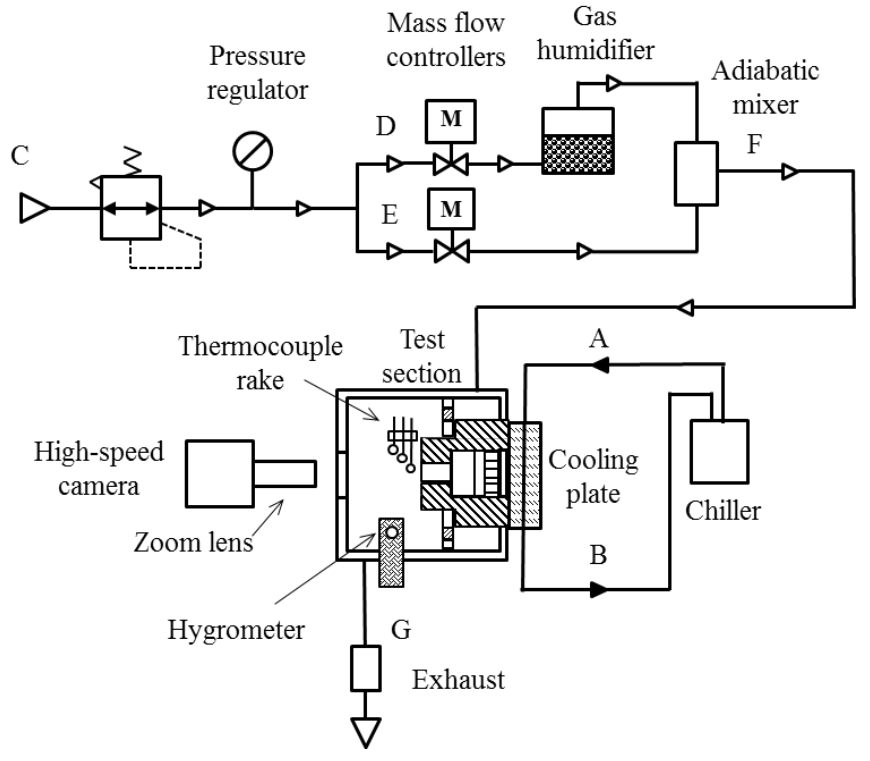

Figure 1. Schematic diagram of nitrogen-water gas mixture and chilled water flow circuits. 


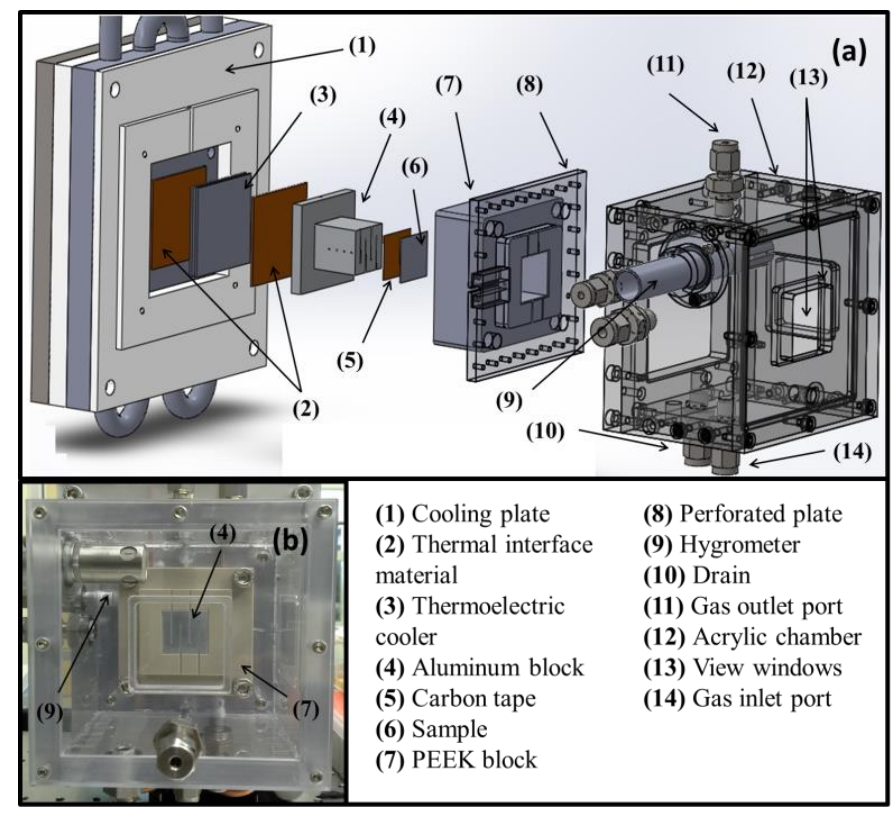

Figure 2. (a) 3D CAD model of the test section exploded assembly, and (b) front view photograph of the chamber. 


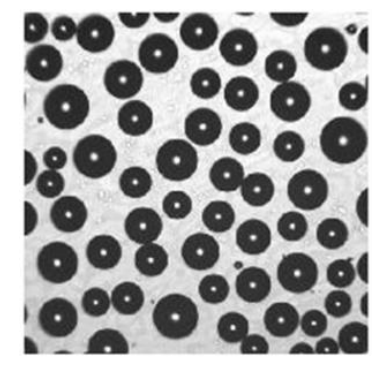

(a)

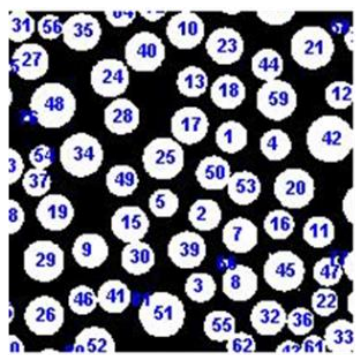

(c)

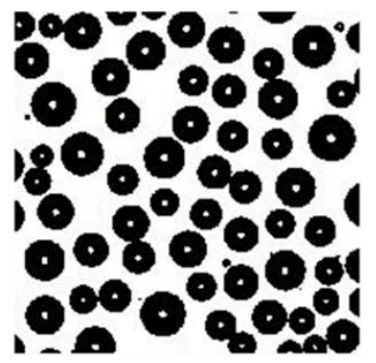

(b)

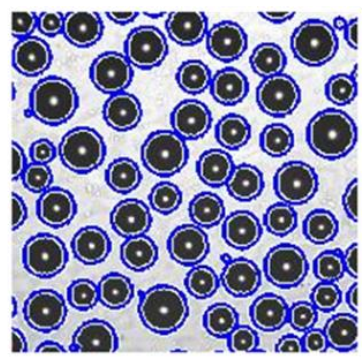

(d)

Figure 3. Example image subject to post-processing by (a) contrast enhancement, (b) binary segmentation, (c) color scheme reversal, elimination of unphysical reflections, and droplet tagging, and (d) circular droplet boundary detection ( $\left.R H=70 \% ; \Delta T_{\text {sub }}=15^{\circ} \mathrm{C}, t=15.5 \mathrm{~min}\right)$. 


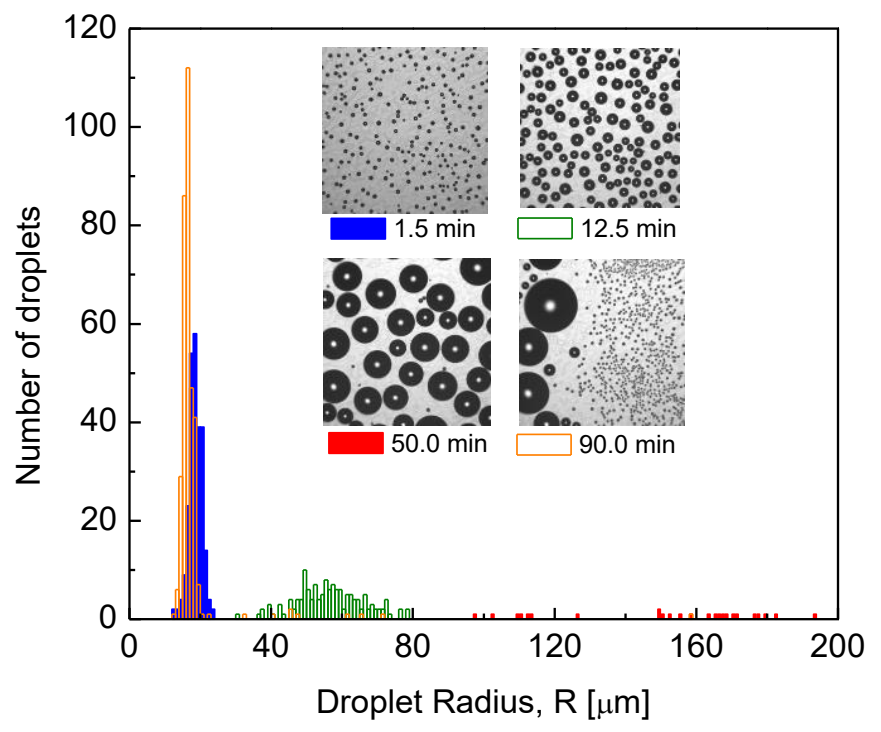

(a)

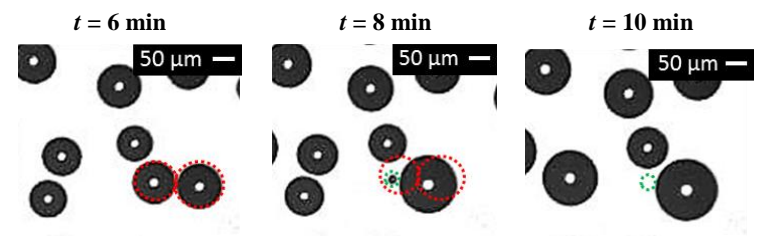

(b)

Figure 4. (a) Histogram of number of droplets at a given size at $t=1.5,12.5,50$, and $90 \mathrm{~min}$. Inset images show condensed droplets on the substrate. (b) The set of bottom images depicts a coalescence event in regime $\left(\ddot{i i}_{B}\right) .\left(R H=70 \% ; \Delta T_{\text {sub }}=15{ }^{\circ} \mathrm{C}\right.$. $)$ 


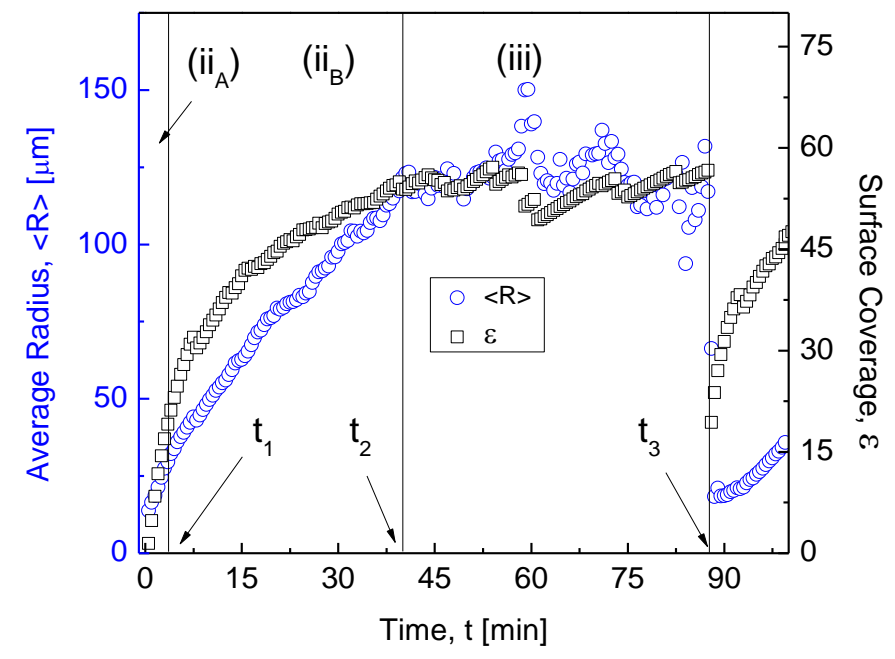

Figure 5. Average radius (left axis) and surface coverage (right axis) versus time for $R H=70 \%$ and $\Delta T_{\text {sub }}=15^{\circ} \mathrm{C}$. 


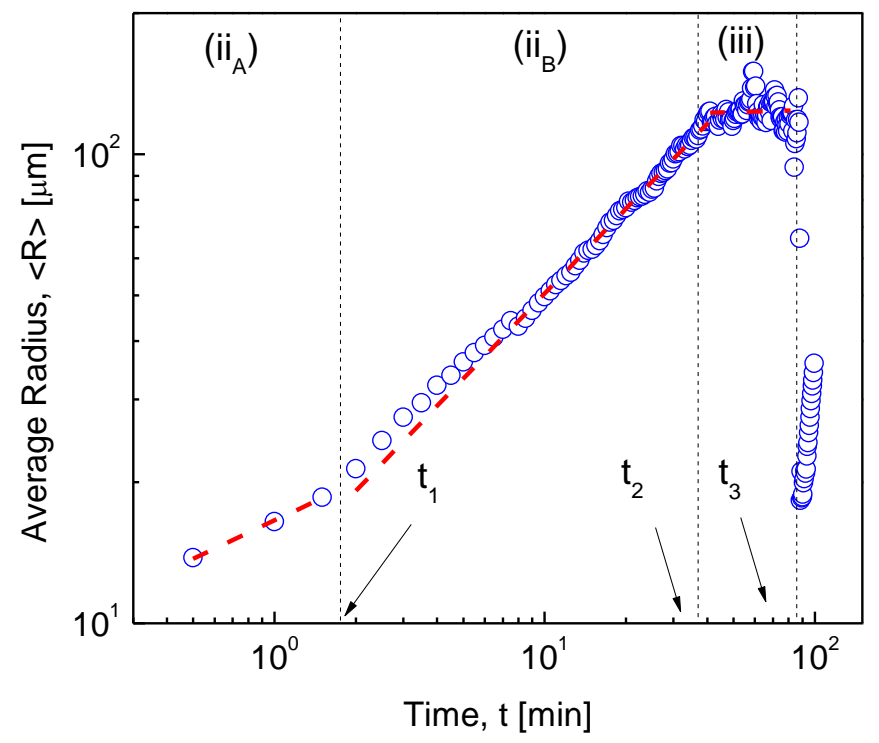

Figure 6. Average radius versus time for $R H=70 \%$ and $\Delta T_{s u b}=15{ }^{\circ} \mathrm{C}$. Dashed lines indicate power-law fits within each regime. 


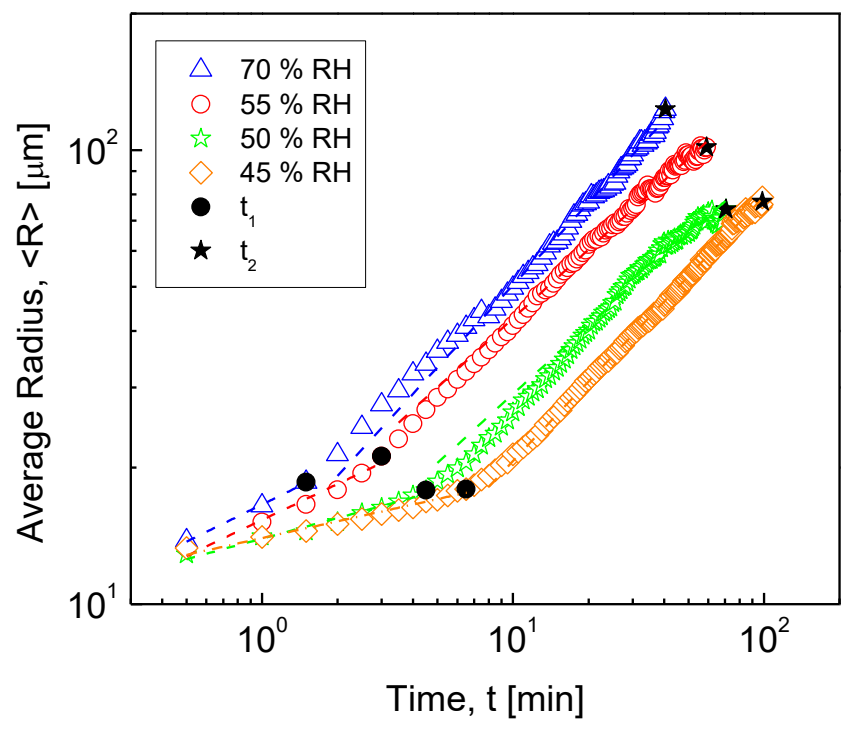

Figure 7. Average radius versus time at $\Delta T_{\text {sub }}=15{ }^{\circ} \mathrm{C}$ and $70 \%, 55 \%, 50 \%$, and $45 \%$ relative humidity. 


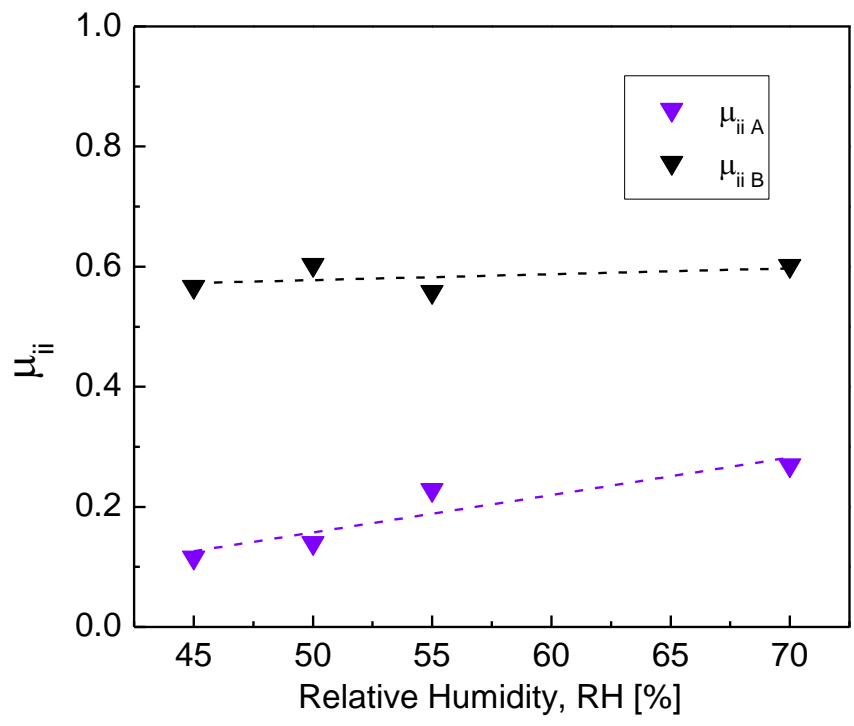

Figure 8. Exponent of the power-law fit to the temporal average radius data of the form $\left\langle R>\sim t^{\mu}\right.$ for regime $\left(i i_{A}\right)\left(\mu_{i i, A}\right)$ and regime $\left(i i_{B}\right)\left(\mu_{i i, B}\right)$ as a function of relative humidity. 


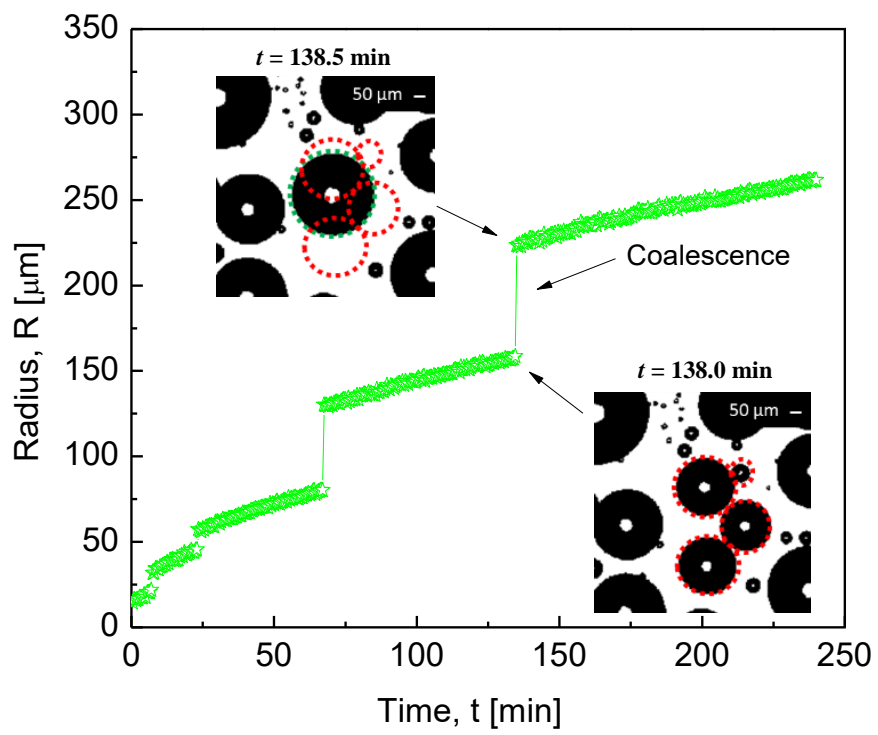

Figure 9. Radius versus time for a single droplet at $\Delta T_{s u b}=15{ }^{\circ} \mathrm{C}$ and $50 \%$ relative humidity. Inset images show a set of droplets before and after the last coalescence event. 


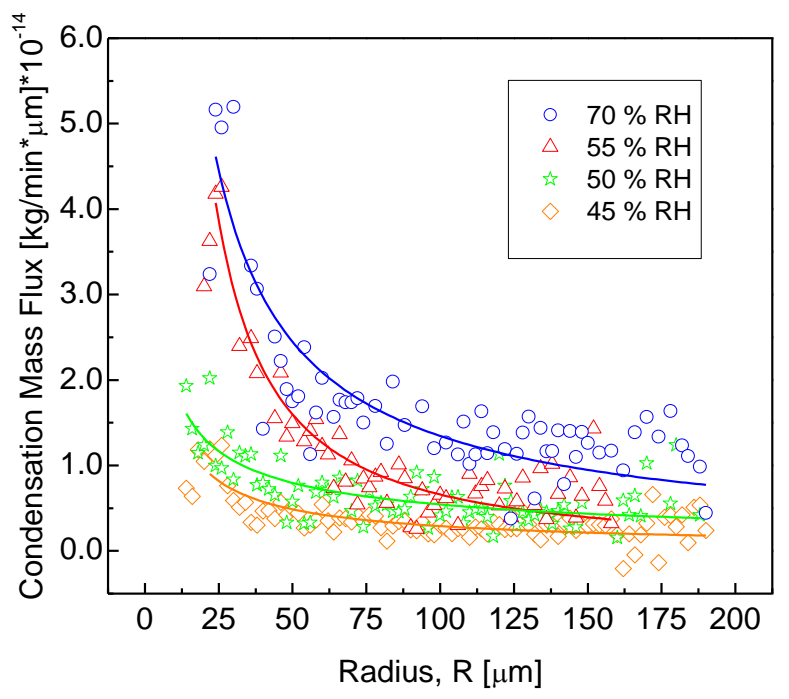

Figure 10. Rate of condensation per unit surface area of a single droplet as a function of droplet radius at $\Delta T_{\text {sub }}=15{ }^{\circ} \mathrm{C}$ and $70 \%, 55 \%, 50 \%$, and $45 \%$ relative humidity. 


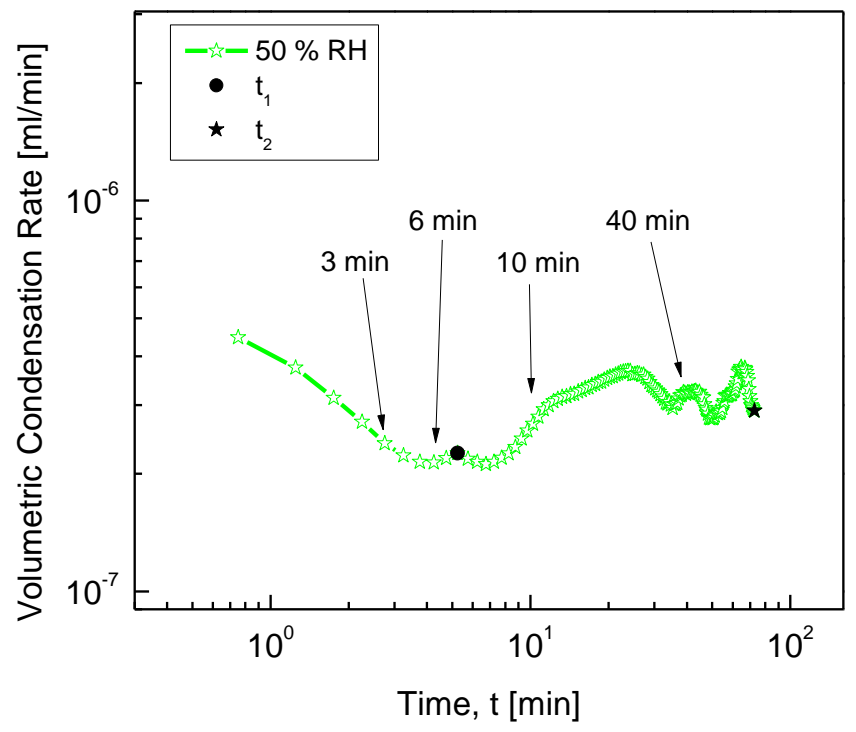

(a)

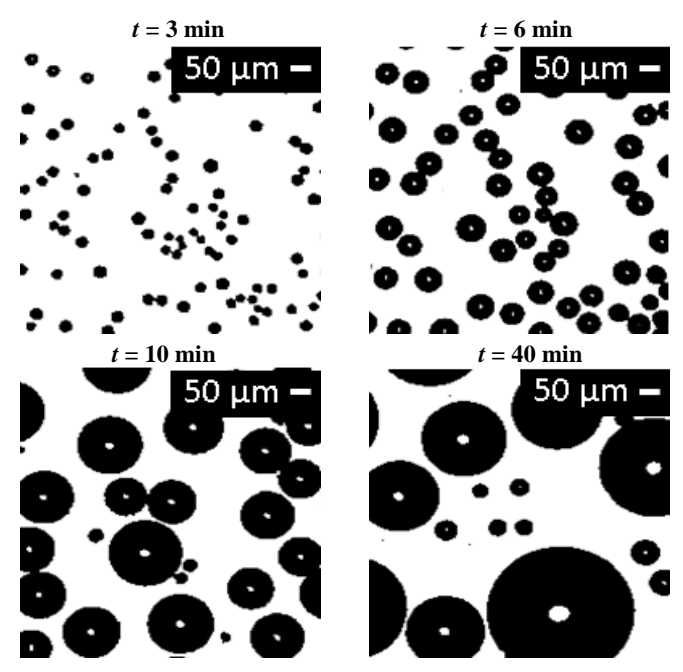

(b)

Figure 11. (a) Volumetric rate of condensation on the surface as function of time, and (b) time series of images of the droplets on the surface for $\Delta T_{\text {sub }}=15^{\circ} \mathrm{C}$ and $50 \%$ relative humidity. 


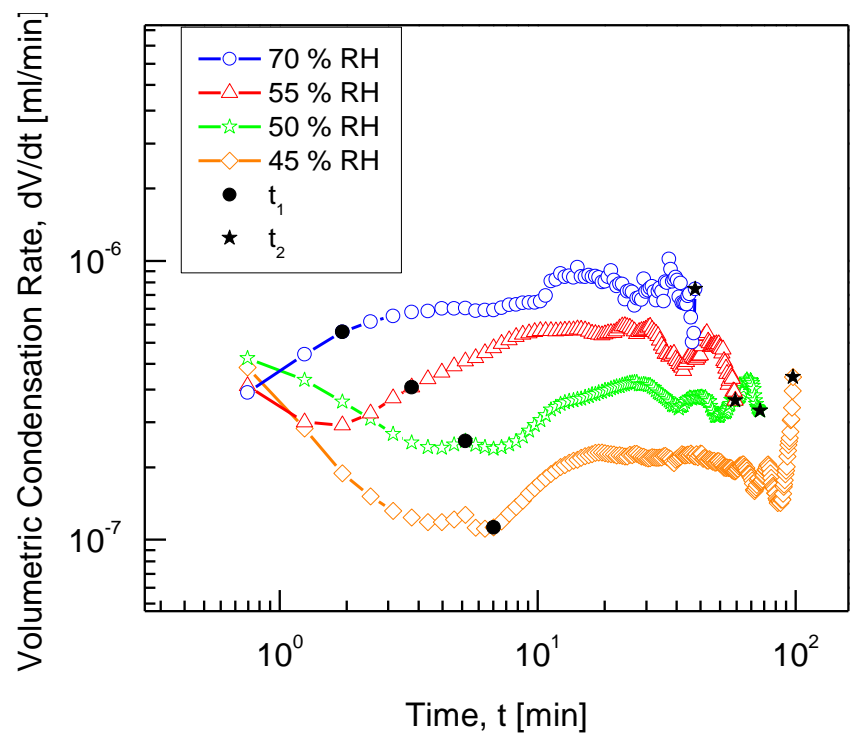

Figure 12. Volumetric rate of condensation on the surface as a function of time at $\Delta T_{\text {sub }}=15{ }^{\circ} \mathrm{C}$ and $70 \%, 55 \%, 50 \%$, and $45 \%$ relative humidity. 